\title{
Assessment and management of rapid-cycling bipolar affective disorder
}

\author{
Mukhtar Ahmed \& Richard Morriss
}

Rapid-cycling bipolar affective disorder presents a unique spectrum of therapeutic difficulties. This article outlines some practical guidelines on the assessment and management of the disorder in the light of current research and clinical practice.

The term 'rapid cycling' was coined by Dunner \& Fieve (1974). They arbitrarily defined rapid cycling as four or more mood episodes per year. Rapid cycling in relation to bipolar affective disorder has been defined by the DSM-IV criteria (American Psychiatric Association, 1994)(Box 1).

Some patients show cycling frequencies in the order of weeks to days, a phenomenon known as ultra-rapid cycling. Cycling of discrete mood phases of less than 24 hours has been described as ultra-ultra rapid cycling or ultra-radian cycling (Kramlinger \& Post, 1996).

\section{Prevalence, demographic characteristics and course}

Rapid cycling has a prevalence of $13-20 \%$ of all out-patients with bipolar disorder presenting to psychiatrists. These prevalence figures are derived largely from patient samples attending tertiary care centres specialising in the treatment of affective disorder, and may be overestimated compared with a British district general hospital.

Rapid- and non-rapid-cyclers are indistinguishable clinically with regard to age at onset, duration of illness, marital status and family history of affective disorders (Coryell et al, 1992). More women than men are rapid-cyclers.

\section{Clinical course variables}

Most studies show that rapid cycling predicts poor prognosis, characterised by a greater number of life-time episodes; a greater likelihood of comorbid psychiatric disorders such as anxiety, substance misuse, eating disorder, and personality disorder; and a poorer response to lithium (Dunner \& Fieve, 1974). However, Coryell et al (1992) found that rapid cycling in the majority of patients is a transient clinical presentation in the longitudinal course of bipolar disorder. In more than $60 \%$ of

Box 1. DSM-IV criteria for rapid-cycling bipolar disorder

At least four episodes of a mood disturbance in the previous 12 months that meet the criteria for a major depressive, manic, mixed or hypomanic episode

Episodes are demarcated by either partial or full remission for at least two months, or a switch to an episode of the opposite polarity* (e.g. major depressive episode to manic episode)

Mood episodes that count towards defining rapid cycling exclude those episodes directly caused by a substance (e.g. cocaine, corticosteroid) or general medical condition

*Manic, hypomanic or mixed episodes are counted as being at the same pole

Mukhtar Ahmed works as a Registrar in Psychiatry on the northern rotation in the North-West Region. He has clinical and research interests in somatisation disorder and bipolar disorder. Richard Morriss, MRCPsych, is a Senior Lecturer and Honorary Consultant in Psychiatry at the University of Manchester and Guild NHS Trust in Preston (Royal Preston Hospital, Sharoe Green Lane, Fulwood, Preston PR2 4HT). His research interests are in psychological treatments of bipolar disorder, somatisation, chronic fatigue syndrome, suicide research and primary care psychiatry. 
their cases, rapid cycling lasted for less than two years. Some reports have indicated that more rapid-cyclers have bipolar II patterns with severe depression but less-severe hypomania.

Rapid cycling emerges relatively late in the course of the affective illness in approximately $80 \%$ of cases but in $20 \%$ of patients it may manifest itself from the outset (Goodwin \& Jamison, 1990). Later rapid cycling could reflect the impact of certain treatments such as antidepressants, or comorbid medical problems such as hypothyroidism (in many instances brought about through lithium therapy) accelerating the natural course of the disorder. The fact that rapid cycling generally develops later in the course of the illness may reflect underlying pathophysiological mechanisms such as kindling or sensitisation (Post et al, 1986) (see Box 2).

In support of the latter theories about pathophysiology, rapid-cycling patients and other difficult-to-treat groups of patients with bipolar disorders often show generalised cerebral white matter changes on magnetic resonance imaging, electroencephalograph (EEG) abnormalities and abnormalities on neuropsychological testing when compared with bipolar patients who respond to conventional mood-stabilising drugs.

Although bipolar disorder is genetically inherited, rapid cycling appears to arise from factors separate from the genetic vulnerability to bipolar illness and which do not lead to aggregation within families (Nurenberger et al, 1988). Research suggests that individuals who experience frequent mood episodes face a greater risk of suicide (Fawcett et al, 1987). It has also been suggested that the swing from mania or hypomania to depression is an important clinical predictor of suicide (Beck et al, 1985).

\section{Effect on patient and family}

Rapid cycling requires assessment and treatment to reduce the cycling frequency for a number of clinical reasons such as the high risk of suicide, the high economic cost to health services of readmission to hospital, and the potentially deleterious effect on the longitudinal course of the patient's illness through kindling and sensitisation. More episodes of illness earlier in the course of bipolar disorder predict more frequent cycling later in the course of illness.

However, the most pressing clinical reason for the recognition, assessment and management of rapid cycling is the devastating effect it has on the patient and family. Rapid cycling usually means that the patient is unable to return to work, family relationships may not recover from the effect of the patient's previous behaviour, and neither the patient nor the family can make any short- or long-
Box 2. Mechanisms derived from animal models to explain the shorter time between relapses longitudinally in some bipolar disorder patients

Kindling -refers to the development of focal electrical seizure in response to repeated intermittent electrical stimulation of the brain with insufficient current. The seizure takes the form of mood disturbance rather than an epileptic seizure. Gradually, there is neuronal damage

Behavioural sensitisation - refers to an increasing behavioural responsivity to repeated, intermittent application of psychomotor stimulants, with evidence of involvement of conditioning to the environment

Both kindling and behavioural sensitisation could result in affective relapses becoming progressively more easily triggered by the same circumstances. Eventually, no triggers are required, and hypomanic or depressive relapses occur spontaneously

term plans in life for fear of future relapses (Vocisano et al, 1996).

\section{Clinical and psychosocial factors}

The following factors should be systematically examined in every patient with rapid-cycling bipolar affective disorder. If these factors can be modified, the patient may cycle less frequently, lead a less disrupted life, and respond to lithium treatment.

\section{Life events and family atmosphere}

Since most rapid cycling occurs later in the course of illness of most bipolar patients, life events and adverse family atmosphere rarely precipitate rapid cycling directly; such factors could, however, induce behaviour such as drinking, illicit drug use or non-adherence with prescribed medications, which in turn precipitate rapid cycling in patients with established bipolar disorder. 


\section{Comorbid alcohol and substance misuse}

It is widely recognised that the coexistence of bipolar disorder and substance misuse increases mortality from suicide, increases relapse rates and predicts poor response to lithium. Data from the Epidemiologic Catchment Area (ECA) study for alcohol misuse and dependence in patients with affective disorders showed a strikingly high lifetime prevalence of $46 \%$ in bipolar patients (Regier et al, 1990) compared with a lifetime risk in the general population of 6-7\%. Patients consistently report that alcohol misuse ameliorates symptom severity. However, alcohol misuse also increases the frequency of relapse in patients with bipolar affective disorder, thereby possibly inducing rapid cycling.

The data on comorbid rates of manic-depressive illness and drug misuse are much less extensive and consistent than those on alcohol misuse. Patients who misuse cocaine and other stimulants or hallucinogenic drugs, including cannabis, are over-represented in samples of bipolar disorder patients. These drugs are used just before manic relapse rather than during a depressive episode. Opiate drugs are not misused by bipolar disorder patients any more than the general population. Although research is scant, most illicit drug misuse appears to increase the frequency of relapse in bipolar affective disorder. Discerning whether substance misuse is a cause (primary) or a complication (secondary) in bipolar patients is often problematic. A number of factors have emerged that are useful in making this distinction but the most important is whether or not substance misuse preceded or followed rapid cycling.

\section{Antidepressants}

There appears to be a group of bipolar patients first presenting with chronic depression followed by antidepressant-induced mania who subsequently develop rapid-cycling bipolar affective disorder if their depression is continually treated with antidepressants (Altshuler et al, 1995). Bipolar patients who have already demonstrated rapid cycling may have further episodes of rapid cycling induced by antidepressants (Bauer et al, 1994). Patients who showed cyclothymia (mild, frequent episodes of mood swings between depression and elation, falling short of the criteria for depressive or hypomanic episodes) before developing treatment-resistant chronic depression are also likely to develop rapid-cycling bipolar disorder if given continuing treatment with antidepressants.
There is no evidence at present, in relation to antidepressants licensed for use in the UK, to suggest that one type of antidepressant is less likely to induce rapid cycling than any other. The antidepressant buproprion, which is not licensed in the UK, may be less likely than tricyclic antidepressants to induce the switch into rapid cycling in patients in the depressive phase of bipolar disorder. There is also no evidence that psychological treatment for depression in bipolar disorder induces mania or rapid cycling. Other bipolar patients with no history of antidepressantinduced mania, no history of cyclothymia preceding a prolonged period of depression, and no previous history of rapid cycling are unlikely to develop rapid cycling as a result of antidepressant use (Box 3). A cautious approach to the withdrawal of antidepressant treatment is warranted in asymptomatic bipolar disorder patients with a history of rapid cycling but rapid withdrawal may be necessary in patients who are currently hypomanic, in a mixed-affective state or in a rapidcycling mood state.

\section{Lithium}

There is a risk that prolonged lithium therapy can induce rapid cycling through the onset of biochemical or symptomatic hypothyroidism. The former is diagnosed if there is persistently raised thyroid-stimulating hormone (TSH) with low or borderline thyroid hormones (T4 or free T4) but no clinical symptoms of hypothyroidism. About $50 \%$ of patients who develop biochemical or symptomatic hypothyroidism while taking lithium carbonate are at an increased risk of developing rapid cycling. These patients require treatment of the thyroid hormone abnormality by either withdrawal or reduction in the dose of lithium treatment, or adding thyroxine (T4). These patients are at a continued risk of rapid cycling through lithium-induced hypothyroidism and may require the long-term prescription of T4 if lithium therapy

Box 3. Factors associated with antidepressantinduced rapid-cycling bipolar disorder

History of antidepressant-induced mania Previous history of rapid cycling

Cyclothymia preceding a period of chronic depression treated continually with antidepressants 
is continued on a long-term basis. Patients who do not develop rapid cycling but show biochemical evidence of hypothyroidism do not require any thyroxine, but thyroid function tests should be checked again in three months' time. Symptomatic hypothyroidism will, of course, require treatment.

There is growing acceptance that rapid lithium withdrawal is associated with an increased risk of immediate relapse into hypomania or depression compared to the expected natural history of bipolar disorder (Goodwin, 1994). However, it is not clear whether rapid lithium withdrawal could induce rapid cycling or whether the recurrence of mania or hypomania upon rapid lithium withdrawal carries a risk for cycle acceleration.

\section{Thyroid abnormalities and other medical conditions}

Of all the potential risk factors for rapid cycling, hypothyroidism has received the most study. Of eight studies, four have suggested that hypothyroidism is more common in rapid- than in non-rapid-cycling bipolar patients (Bauer et al, 1990). All eight studies failed to control for lithium therapy, gender or age, making interpretation of the results difficult. There is also some evidence that peripheral and central thyroid function is controlled separately, so that peripheral measures may not be a reflection of the central function. Although the outcome of research is inconsistent, clinical consensus outlined in the DSM-IV criteria is that even biochemical evidence of hypothyroidism can induce rapid cycling. However, correction of biochemical hypothyroidism is not always effective in rectifying rapid cycling. Other maintaining or precipitating factors for rapid cycling should then be sought, such as substance misuse, non-compliance with mood-stabilising drugs, antidepressant use or a number of other medical disorders (see Box 4). Sometimes no cause can be found and a change in psychotropic medication is required instead.

\section{Treatment strategies}

Management of rapid-cycling bipolar disorder follows the management strategies generally applied to bipolar disorder. Making an accurate diagnosis and assessment of the current episode provides a basis for selecting and staging treatment modalities. Knowledge of the patient's longitudinal pattern of illness is perhaps the most useful guide in planning treatment. Life charts may be of use to delineate the course and pattern of the illness
(Post et al, 1988). Evidence for the effectiveness of medication generally comes from uncontrolled open studies or 'mirror image' studies (a type of before-and-after treatment study) rather than from randomised controlled trials (RCTs).

\section{Lithium carbonate}

Longitudinal research has shown that lithium therapy remains a cornerstone in bipolar disorder prophylaxis as well as having acute antidepressant (in combination with antidepressants) and antimanic effects. However, research has consistently shown that rapid-cycling bipolar disorder predicts poor response to lithium therapy prophylaxis. Dunner \& Fieve (1974) found that $72-82 \%$ of their rapid-cycling patients showed poor response to lithium prophylaxis. This has been replicated by Kukopulos et al (1980) who found that $72 \%$ of their rapid-cycling patients are refractory to lithium prophylaxis. Lithium carbonate, especially in combination with antipsychotic or other moodstabilising agents, can be useful for stabilising rapid-cycling patients in the acute manic, mixedaffective or ultra-rapid cycling phases. However, higher doses with a plasma concentration close to $1.0 \mathrm{mmol} / 1$ may be required but frequent lithium monitoring is necessary and admission to hospital should be considered. Such a treatment strategy is generally not advisable on a long-term basis.

\section{Carbamazepine}

Carbamazepine is prophylactic in bipolar disorder as well as having acute antimanic and possibly acute antidepressant effects in bipolar affective disorder. The most important indication for its use is lithium therapy failure and/or intolerance to side-effects of lithium. Research findings on the use of carbamazepine in rapid-cycling bipolar disorder are inconsistent. Post et al (1987) suggested that rapid cycling, mixed mania and family history of mood disorder were associated with carbamazepine response. Okuma (1993), on the other hand, found that bipolar patients who were rapid cycling or had a history of rapid cycling with continuous recurrence responded unfavourably to lithium or carbamazepine. Research suggests that rapid-cycling patients treated with carbamazepine exhibit an acute response rate of $32 \%$ for depression and $52 \%$ for mania. The prophylaxis response rate for carbamazepine in rapid-cycling patients is $57 \%$ for depression and $59 \%$ for mania. Lithium and carbamazepine prescribed together may be effective in some cases and some authorities recommend the use of this 
Box 4. Medical conditions associated with rapid cycling

Thyroid hypofunction

Graves's disease (hyperthyroidism)

Subarachnoid haemorrhage

Stroke

Multiple sclerosis

Head injury

Gonadal steroid (e.g. oestrogen)

Drugs - e.g. propranolol, levodopa, cyproheptadine

combination from the outset. When lithium and carbamazepine are used together, with or without a neuroleptic, there is a risk of neurotoxicity (tremor, ataxia, nystagmus, altered conscious level, disorientation and muscular fasciculation). A limitation in carbamazepine use over the long term is the loss of prophylactic effect in some patients (termed in the literature 'escape'). Post et al (1990), in a four-year follow-up study of carbamazepine prophylaxis in refractory affective disorder, observed that patients who showed the greatest acceleration in episodes before carbamazepine treatment were the ones who showed the pattern of escape.

\section{Valproate}

Calabrese et al (1993), in a prospective naturalistic open study, demonstrated the acute and the prophylactic effect of sodium valproate in a homogenous group of rapid-cycling patients who received either sodium valproate or sodium valproate with lithium carbonate. A number of other studies with a similar design confirm these findings (e.g. McElroy et al, 1988) but there are no RCTs of sodium valproate in rapid-cycling bipolar disorder patients. Sodium valproate appears to have marked acute and prophylactic antimanic effects, but only mild to moderate acute and prophylactic antidepressant effects. Predictors of acute and prophylactic response to sodium valproate in rapid-cycling bipolar disorder are the absence of psychotic features, absence of borderline personality disorder, presence of mild to moderate hypomania or mixed-affective symptoms. There are RCTs demonstrating the acute effect of sodium valproate on hypomania, but there are no RCTs of sodium valproate prophylaxis in rapid-cycling disorder or bipolar disorder in general.

Recent open studies on the acute treatment of treatment-resistant rapid-cycling bipolar disorder in tertiary referral centres in the USA have demonstrated the effectiveness of a combination therapy of sodium valproate with lithium carbonate. Long-term data on this combination are not yet available. Some authorities advocate the use of carbamazepine and sodium valproate together, or even carbamazepine, sodium valproate and lithium therapy. There are practical difficulties in obtaining satisfactory blood levels of sodium valproate and carbamazepine, since changes in the dosage of one drug generally will affect the level of the other. Sodium valproate levels do appear to correlate with acute antimanic response and there is also a threshold effect.

Few patients benefit from treatment unless a sodium valproate level of $\geq 50 \mu \mathrm{g} / \mathrm{ml}$ is reached. We would recommend that treatment with sodium valproate is initiated at a dose of $250 \mathrm{mg}$ twice or three times daily to reduce gastrointestinal and central nervous system side-effects. The dose is increased by $250-500 \mathrm{mg}$ every three days, according to therapeutic response and side-effects, aiming to achieve a serum concentration of between 50 and $100 \mu \mathrm{g} / \mathrm{ml}$ with a maximum adult dose of $60 \mathrm{mg} / \mathrm{kg}$ per day (monitoring full blood count, liver function and coagulation parameters).

\section{Lamotrigine, gabapentin and other anticonvulsants}

Preliminary open trial and case report data suggest that lamotrigine alone, or in combination with other anticonvulsants or lithium therapy, may have acute and continuing mood-stabilising effects in treatment-resistant rapid- and non-rapid-cycling bipolar patients. There is no evidence concerning its prophylactic effect. In using the combination of lamotrigine and sodium valproate the dosage of lamotrigine should be reduced by half. There are case reports of the successful use of clonazepam to obtain acute stability in rapid-cycling bipolar patients, but there is no evidence of its effectiveness over the long term. Anecdotally, clinicians report some mood-stabilising effect of gabapentin in treatment-resistant rapid-cycling bipolar disorder. There is no evidence that phenytoin, phenobarbitone or chlormethiazole have any clinical effect on rapid-cycling or any other bipolar disorder patients.

\section{Thyroid hormones}

Preliminary data indicated that T4 supplementation may be useful in augmenting the prophylactic efficacy of mood stabilisers in partial responders and rapid-cyclers with bipolar disorder (Whybrow, 
1994). Research suggests that mood stabilisers augmented with enough $\mathrm{T} 4$ to raise the free thyroxine index to approximately 1.5 times the upper limit of the normal range are likely to produce a good therapeutic response.

Pre-existing thyroid function abnormalities do not necessarily predict the response to thyroid hormone supplementation when they are used to augment the mood-stabilising effect of anticonvulsants. There is no evidence to suggest that thyroid supplements prescribed alone are effective in the treatment of rapid-cycling bipolar disorder.

\section{Clozapine and other antipsychotics}

The current licensed clinical indication of clozapine is for treatment-resistant schizophrenia. Recent case reports suggest that clozapine alone, or in combination with lithium, may have acute mood-stabilising properties in rapid-cycling bipolar disorder patients, with or without psychotic features, compared with treatment with mood stabilisers, neuroleptics and thyroid hormones (Calabrese, 1991; Suppeset al, 1994; Frye, 1996). It is suggested that action of clozapine at dopamine $\mathrm{D}_{4}$ receptors, which occur in high density in the limbic region, supports a potential moodstabilising property. Anecdotally, we have also received reports of the effectiveness of risperidone and olanzepine in treatment-resistant rapid-cycling bipolar disorder patients. Many patients who respond to neuroleptics and atypical antipsychotic agents display mood incongruent psychotic symptoms, although neuroleptics are also useful in combination with mood-stabilising drugs in ultrarapid cycling. Patients with rapid-cycling mood disorder with mood-incongruent psychotic features probably require antipsychotic agents in the acute phase of treatment. However, it is not always true that bipolar patients with mood-incongruent psychotic features who acutely respond to antipsychotics require longer-term antipsychotic treatment (unlike patients with schizoaffective disorders who have schizophrenia symptoms, sometimes without prominent mood symptoms). Any conclusions should be drawn with caution since these are case reports or small case series of atypical bipolar disorder. It is possible that all the clinical responses are due to a placebo effect or spontaneous remission.

\section{Verapamil and other calcium channel blockers}

Research on the use of calcium channel blockers in patients unresponsive to lithium or other mood stabilisers is scant, with methodological problems and mixed results. One randomised, placebocontrol study of verapamil reported no therapeutic benefit. There is a report from a preliminary controlled double-blind study suggesting that nimodipine may be effective in rapid-cycling bipolar disorder (Pazzaglia et al, 1995).

\section{Conclusion}

Rapid-cycling bipolar disorder is rare but is difficult to treat and has a high rate of suicide. It causes considerable disruption to the life of the patient and their family. Fortunately, in more than $50 \%$ of cases, rapid cycling lasts for less than two years. It generally develops late in the course of bipolar disorder. Often, rapid cycling can be managed by the correct identification and management of a small number of comorbid medical problems, substance misuse and treatment variables. Rapid cycling is relatively resistant to lithium treatment, but has a better response to carbamazepine and sodium valproate. Sometimes, symptomatic control requires combinations of a number of mood stabilisers with or without an antipsychotic. Definitive evidence from RCTs of the effectiveness of these mood-stabilising agents in rapid-cycling patients is not yet available.

\section{References}

Altshuler, L. L., Post, R. M., Leverich, G. S., et al (1995) Antidepressant-induced mania and cycle acceleration: a controversy revisited. American Journal of Psychiatry, 152, 1130-1138.

American Psychiatric Association (1994) Diagnostic and Statistical Manual of Mental Disorders (4th edn) (DSM-IV). Washington, DC:APA.

Bauer, M. S., Whybrow, P. C. \& Winkur, A. (1990) Rapid-cycling bipolar affective disorder. I. Association with grade 1 hypothyroidism. Archives of General Psychiatry, 47, 427-432.

-, Calabrese, J., Dunner, D. L., et al (1994) Multisite data reanalysis of the validity of rapid cycling as a modifier for bipolar disorder in DSM-IV. American Journal of Psychiatry, 151, 506-515.

Beck, A. T., Steer, R. A., Kovacs, M., et al (1985) Hopelessness and eventual suicide: $A 10$ year prospective study of patients hospitalised with suicidal ideation. American Journal of Psychiatry, 142, 559-560.

Calabrese, J. R. (1991) Clozapine prophylaxis in rapid-cycling bipolar disorder. Journal of Clinical Psychopharmacology, 11, 396-397.

-, Woyshiville, M. J., Kimmel, S. E., et al (1993) Predictors of valproate response in bipolar rapid cycling. Journal of Clinical Psychopharmacology, 13, 280-283.

Coryell, W., Endicott, J. \& Keller, M. (1992) Rapid cycling affective disorder: demographics, diagnosis, family history, and course. Archives of General Psychiatry, 49, 126-131.

Dunner, L. \& Fieve, R. R. (1974) Clinical factors in lithium carbonate prophylaxis failure. Archives of General Psychiatry, 30, 229-233. 
Fawcett, J., Scheeftner, W., Cark, D., et al (1987) Clinical predictors of suicide in patients with major affective disorders: a controlled prospective study. American Journal of Psychiatry, 44, 35-40.

Frye, M. A. (1996) Clozapine in rapid cycling bipolar disorder. Journal of Clinical Psychopharmacology, 16, 87-90.

Goodwin, F. K. \& Jamison, K. R. (1990) Manic-Depressive Illness. New York: Oxford University Press.

Goodwin, G. M. (1994) Recurrence of mania after lithium withdrawal. Implications for the use of lithium in the treatment of bipolar affective disorder. British Journal of Psychiatry, 164, 149-152.

Kramlinger, K. \& Post, R. (1996) Ultra-rapid and ultradian cycling in bipolar affective illness. British Journal of Psychiatry, 168, 314-323.

Kukopulos, A., Reginaldi, D., Laddomada, P., et al (1980) Course of manic depressive cycle and course caused by treatment. Pharmacopsychiatry, 13, 156-167.

McElroy, S. L., Keck, P. E., Pope, H. G., et al (1988) Valproate in the treatment of rapid-cycling bipolar disorder. Journal of Clinical Psychopharmacology, 8, 275-279.

Nurenberger, J., Jr, Guroff, J. J., Hamovit, J., et al (1988) A family study of rapid-cycling bipolar illness. Journal of Affective Disorders, 15, 87-91.

Okuma, T. (1993) Effect of carbamazepine and lithium on the affective disorder. Neuropsychobiology, 27, 138-145.

Pazzaglia, P. J., Post, R. M., Ketter, T. A., et al (1995) Preliminary controlled trial of nimodipine in ultra-rapid cycling affective dysregulation. Psychiatry Research, 49, 257-272.

Post, R., Rubinow, D. R. \& Ballinger, J. C.(1986) Conditioning and sensitisation in the longitudinal course of affective disorder. British Journal of Psychiatry, 149, 191-201.

—,Uhde, T. W., Roy-Byrne, P. P., et al (1987) Correlates of antimanic response to carbamazepine. Psychiatry Research, 21, 71-83.

—, Roy-Burne, P. P. \& Uhde, T. W. (1988) Graphic representation of the life course of illness in patients with affective disorder. American Journal of Psychiatry, 145, 844-848.

-, Leverich G. S., Rossof, A. S., et al (1990) Carbamazepine prophylaxis in refractory affective disorder: a focus on longterm follow-up. Journal of Clinical Psychopharmacology, 10, 318327.

Regier, D. A., Farmer, M. E., Rae, D. S., et al (1990) Comorbidity of mental disorders with alcohol and other drug abuse: results from the Epidemiological Catchment Area (ECA) study. Journal of the American Medical Association, 264, 2517-2518.

Suppes, T., Phillips, K. A. \& Judd, C. R. (1994) Clozapine treatment of nonpsychotic rapid cycling bipolar disorder: a report of three cases. Biological Psychiatry, 36, 338-340.

Vocisano, C., Klein, D. N., Keefe, R. S. E., et al (1996) Demographic, family history, premorbid functioning, developmental characteristic, and course of patients with deteriorated affective disorder. American Journal of Psychiatry, 153, 248-255.

Whybrow, P. C. (1994) The therapeutic use of triiodothyronine and high dose thyroxine in psychiatric disorder. Acta Medica Austrica, 21, 47-52.

\section{Multiple choice questions}

1. Rapid-cycling bipolar disorder is a term which is restricted to:

a patients who cycle between hypomania and depression each day

b patients who cycle between hypomania and depression each week

c patients with features of depression and hypomania at the same time d patients who have four or more episodes of depression or hypomania each year

e patients with mood-incongruent psychotic features as well as hypomania or depression.

2. Rapid-cycling bipolar disorder:

a usually presents for life once it appears

b lasts less than two years in $50 \%$ of cases

c usually occurs late in the longitudinal course of bipolar disorder

$\mathrm{d}$ is genetically inherited in families with multiply affected individuals with rapidcycling bipolar disorder

e occurs in men more often than women.

3. Known risk factors for developing rapidcycling mood disorder include:

a history of antidepressant-induced hypomania

b substance misuse

c biochemical evidence of low thyroxine levels

d a recent severe life event

e lithium therapy-induced hypothyroidism.

4. Acute treatment of rapid-cycling mood disorder is often effective with:

a lithium therapy alone

b sodium valproate alone in the hypomanic phase

c sodium valproate and lithium therapy

d SSRIs in the depressed phase

e selective serotonin reuptake inhibitors in ultra-rapid cycling.

5. Prophylactic treatment of rapid-cycling mood disorder is often effective with:

a carbamazepine alone

b haloperidol alone

c lithium carbonate alone

d mood stabilising drugs and high doses of L-thyroxine

e sodium valproate alone.

\begin{tabular}{|c|c|c|c|c|}
\hline \multicolumn{5}{|c|}{ MCQ answers } \\
\hline 1 & 2 & 3 & 4 & 5 \\
\hline a $F$ & a $F$ & a $T$ & a $F$ & a $T$ \\
\hline b F & b $\mathrm{T}$ & b $T$ & b $T$ & b F \\
\hline c $F$ & c $\mathrm{T}$ & c $\mathrm{T}$ & c $T$ & c $\mathbf{F}$ \\
\hline d $\mathrm{T}$ & d F & d F & d $T$ & d $\mathrm{T}$ \\
\hline e $F$ & e $F$ & e $T$ & e $F$ & e $T$ \\
\hline
\end{tabular}

